\title{
Isolated metachronous splenic metastasis from synchronous colon
}

\section{cancer}

\author{
Rasim Gencosmanoglu*1,2, Fugen Aker ${ }^{3}$, Gozde Kir ${ }^{4}$ and Nurdan Tozun 5
}

Address: ${ }^{1}$ Department of General Surgery, Marmara University School of Medicine, Istanbul, Turkey, ${ }^{2}$ Unit of Surgery, Marmara University Institute of Gastroenterology, Istanbul, Turkey, ${ }^{3}$ Department of Pathology, Haydarpasa State Hospital, Istanbul, Turkey, ${ }^{4}$ Department of Pathology, Umraniye State Hospital, Istanbul, Turkey and ${ }^{5}$ Department of Gastroenterology, Marmara University School of Medicine, Istanbul, Turkey

Email: Rasim Gencosmanoglu* - rgencosmanoglu@marmara.edu.tr; Fugen Aker - fugenaker@yahoo.com; Gozde Kir - gozkir@yahoo.com; Nurdan Tozun - nurdantozun@marmara.edu.tr

* Corresponding author

Published: 06 July 2006

World Journal of Surgical Oncology 2006, 4:42 doi:10.1 186/1477-7819-4-42

This article is available from: http://www.wjso.com/content/4/I/42

(C) 2006 Gencosmanoglu et al; licensee BioMed Central Ltd.

This is an Open Access article distributed under the terms of the Creative Commons Attribution License (http://creativecommons.org/licenses/by/2.0), which permits unrestricted use, distribution, and reproduction in any medium, provided the original work is properly cited.

\begin{abstract}
Background: Isolated splenic metastases from colorectal cancer are very rare and there are only 13 cases reported in the English literature so far. Most cases are asymptomatic and the diagnosis is usually made by imaging studies during the evaluation of rising CEA level postoperatively.

Case presentation: A 76-year-old man underwent an extended left hemicolectomy for synchronous colon cancers located at the left flexure and the sigmoid colon. The tumors were staged as IIIC (T3N2M0) clinically and the patient received adjuvant chemotherapy. During the first year follow-up period, the patient remained asymptomatic with normal levels of laboratory tests including CEA measurement. However, a gradually rising CEA level after the $14^{\text {th }}$ postoperative month necessitated further imaging studies including computed tomography of the abdomen which revealed a mass in the spleen that was subsequently confirmed by ${ }^{8}$ FDG- PET scanning to be an isolated metastasis. The patient underwent splenectomy 17 months after his previous cancer surgery. Histological diagnosis confirmed a metastatic adenocarcinoma with no capsule invasion. After an uneventful postoperative period, the patient has been symptom-free during the one-year of follow-up with normal blood CEA levels, although he did not accept to receive any further adjuvant therapy. To the best of our knowledge, this $14^{\text {th }}$ case of isolated splenic metastasis from colorectal carcinoma is also the first reported case of splenic metastasis demonstrated preoperatively by ${ }^{18}$ FDG PET-CT fusion scanning which revealed its solitary nature as well.
\end{abstract}

Conclusion: Isolated splenic metastasis is a rare finding in the follow-up of colorectal cancer patients and long-term survival can be achieved with splenectomy.

\section{Background}

Splenic metastases from colorectal carcinoma are very rare and usually occur as a component of disseminated disease. From the first case report of isolated metastasis to the spleen in the setting of colorectal carcinoma by Dunbar $e t$ al. [1] in 1969, only thirteen cases are reported in the Eng- lish literature to date [2-13]. Twelve of them were metachronous metastases, whereas the remaining one synchronous. Although the exact incidence of splenic metastases is still unknown, Berge [14] reported the overall incidence of splenic involvement as $7.1 \%$ in 7165 autopsy cases with various cancers and the incidence of 
splenic metastasis from colon and rectal carcinomas as $4.4 \%$ and $1.6 \%$, respectively. However, he did not report any case of solitary splenic metastasis. Anatomical, histological and functional features of spleen have been speculated as the reason of the rarity for solitary cancer metastasis [15]. Most cases are reported as asymptomatic and the diagnosis is usually made by the imaging studies such as abdominal ultrasound (US) or computerized tomography (CT) during the evaluation of a rising CEA level in the postoperative follow-up period of colorectal cancer patients. To the best of our knowledge, this is the first case in which the splenic metastasis from colorectal carcinoma was demonstrated preoperatively by ${ }^{18}$ FDG PET-CT fusion scanning which also revealed its solitary nature.

\section{Case presentation}

A 76-year-old man suffering from fatigue, weight loss and rectal bleeding underwent extended left hemicolectomy with the diagnosis of synchronous colon cancer. Preoperative work-up including colonoscopy and contrastenhanced abdominal computed tomography (CT) showed that the tumors located at the left flexure and the sigmoid colon and the endoscopic biopsies revealed the diagnosis of adenocarcinoma for both of the tumors. There was neither liver nor other remote site metastasis in the abdomen. The chest X-ray was normal. However, CEA level was higher (34.63 ng/mL, Reference values: 0-10 ng/ $\mathrm{mL}$ ) in the preoperative period (Figure 1). Histopathological examination of the specimen revealed a moderately differentiated adenocarcinoma, $2 \mathrm{~cm}$ in diameter, that invaded into but not beyond the muscularis propria (pT2) at the left flexure with lymphatic and vascular but not perineural invasion and another moderately differentiated adenocarcinoma, $6 \mathrm{~cm}$ in diameter, that invaded into the serosa (pT3) with lymphatic, vascular and perineural

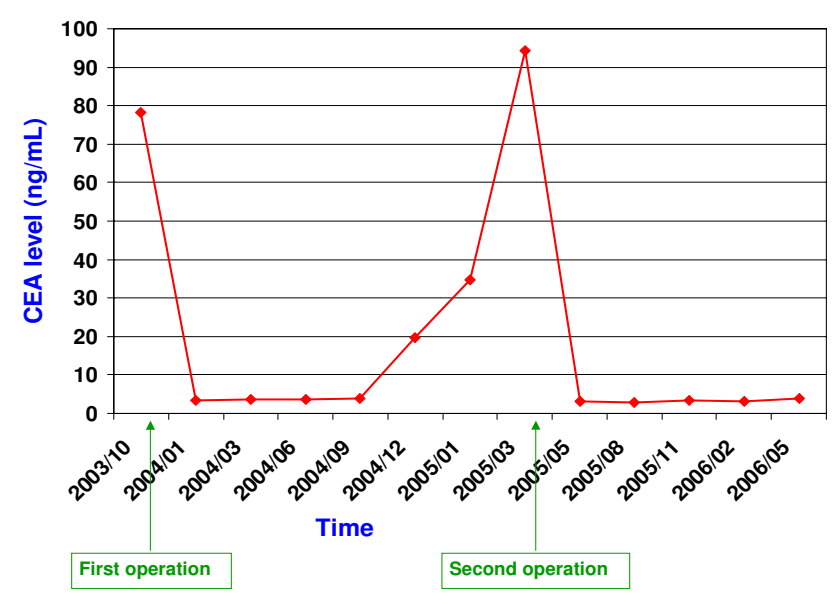

\section{Figure I}

The course of pre- and post-operative blood CEA levels (Reference values: 0-10 ng/mL). invasion. Both of the tumors had similar histologic features that were well differentiated and complex glands composed of stratified columnar cells with eosinophilic cytoplasms accompanied by large necrotic areas. Four of the nine lymph nodes were positive for metastatic adenocarcinoma with the presence of perinodal invasion ( $\mathrm{pN} 2$ ). Therefore, the patient was classified as stage $\mathrm{C} 2$ according to the modified Astler-Coller staging system and stage IIIC (pT3N2, cT3N2M0) according to the TNM system. He received oral chemotherapy consisting of capecitabine (Xeloda ${ }^{\circledR}$, Roche) $5 \times 500 \mathrm{mg}$ capsule for 14 days with 7day interval for a total of 8 courses. Blood CEA level decreased to the $3.54 \mathrm{ng} / \mathrm{mL}$ in the early postoperative period and remained normal up to the $14^{\text {th }}$ month of follow-up. A rising CEA level (Figure 1) in this asymptomatic case necessitated further imaging studies including abdominal ultrasound and contrast-enhanced abdominal and thoracic CT which revealed a hyperdense solid mass in the lower two third of the spleen, while the liver and the paraaortic area were disease-free. Furthermore, 18FDGPET scanning showed an isolated hypermetabolic state in the spleen (Figure 2A), while PET-CT fusion scanning confirmed this pathological FDG uptake as superimposing with the hypodense mass in the spleen (Figure 2B). Any other remote organ metastases were not found by both CT and PET scanning. Following a preoperative vaccination against Streptococcus pneumoniae, Haemophilus influenzae, and Neisseria meningitidis as prevention strategy to avoid overwhelming postsplenectomy infection, the patient underwent splenectomy 17 months after his previous colorectal cancer surgery. At laparotomy, the tumor was in the lower two third of the spleen without any capsule invasion macroscopically (Figure 3 ) and there were neither liver nor other intraabdominal organ metastases. Also, any lymph node involvement at splenic hilus or paraaortic site was not detected during the operation. Therefore, lymphadenectomy at either site was not performed. Histopathologic examination of the splenectomy specimen showed that the tumor within the spleen was a metastasis of a moderately differentiated adenocarcinoma with $5 \times 6 \times 6.5 \mathrm{~cm}$ in dimensions and it was sharply demarcated from the adjacent splenic parenchyma without any capsule invasion (Figure 4A). The histologic features of this metastatic tumor were very similar to the primary synchronous colonic adenocarcinomas (Figure $4 \mathrm{~B})$. Because the patient had a previous colon cancer surgery and no findings of any primary tumor (i.e. adenocarcinoma) in any other organ except metastatic splenic tumor at laparotomy as well as on the preoperative CT and PET scanning besides the decrease of the high blood CEA level to within normal limits following splenectomy and the presence of histologic similarities between the metastatic tumor and the primaries, the present case was accepted as a solitary metachronous splenic metastasis from colon cancer. The postoperative period was unevent- 


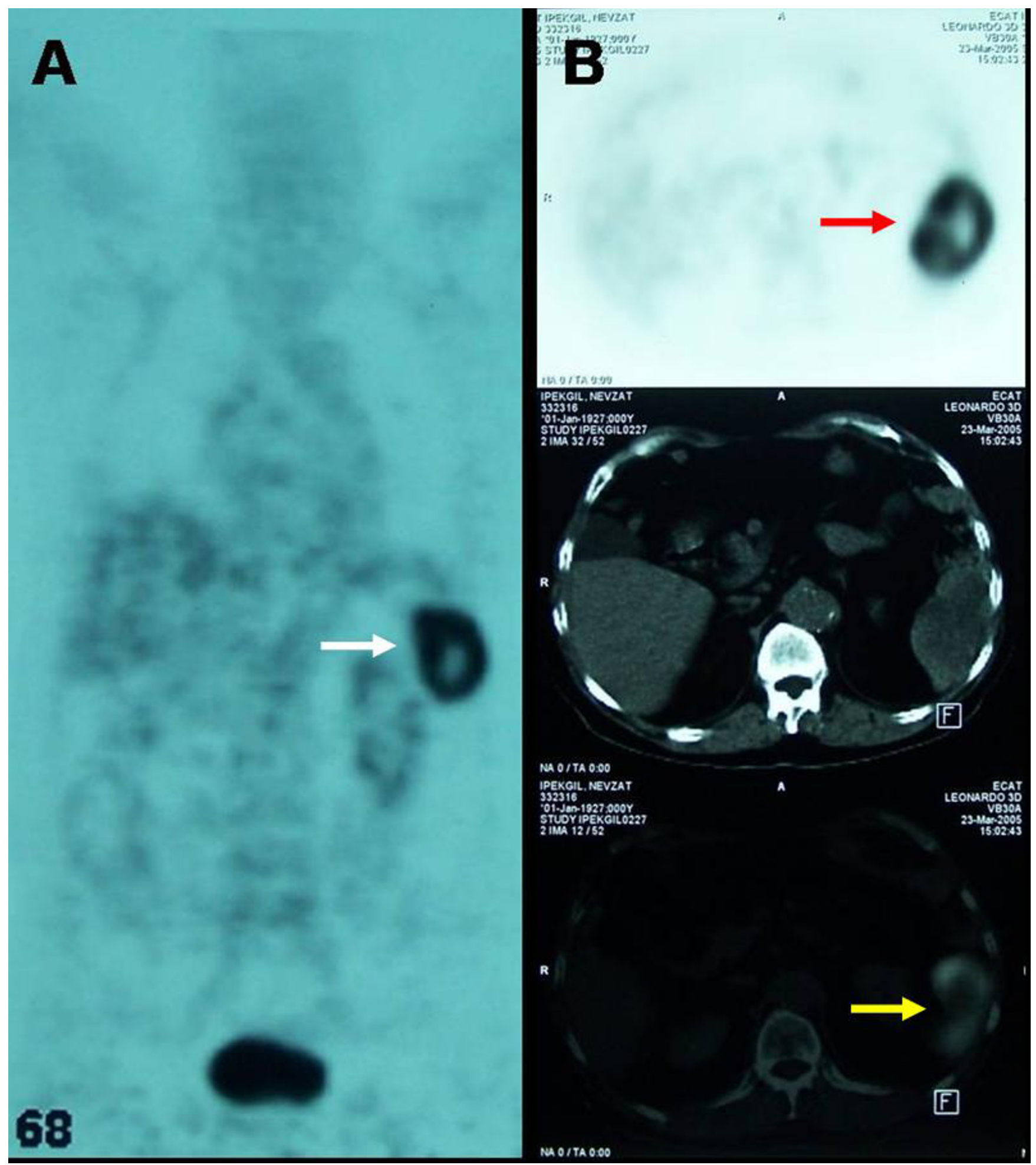

Figure 2

I8FDG- PET scanning showed an isolated hypermetabolic state in the spleen (white arrow) (A). PET-CT fusion scanning confirmed that this pathological FDG uptake (red arrow) superimposed with the hypodense mass in the spleen (yellow arrow) (B). 


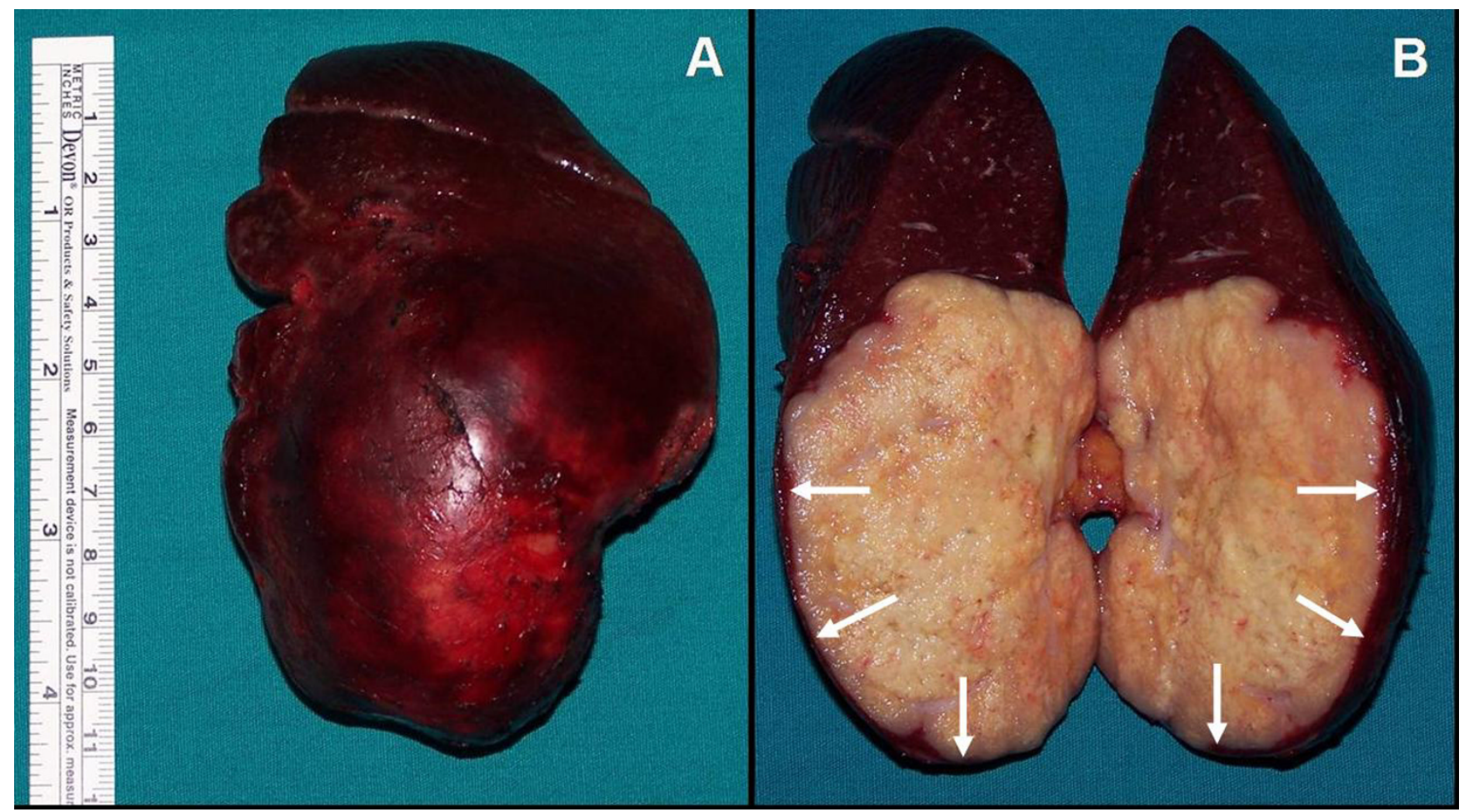

Figure 3

The macroscopic view of the splenectomy specimen $(A)$ and its cross section (B). Note that the splenic capsule was not invaded by the metastatic tumor (arrows).

ful. The patient has been symptom-free during the oneyear of follow-up with a normal blood CEA levels, although he did not accept to receive any further adjuvant therapy.

\section{Discussion}

Several theories have been postulated for the reason of the rarity of solitary splenic metastasis from colorectal cancer. Although the incidence of micrometastases in the spleen secondary to colorectal carcinoma is reported as $4.4 \%$ in careful autopsy examinations [14], the rare occurrence of clinically apparent metastasis may propose the existence of a certain mechanism prohibiting tumor cell proliferation in the spleen. The sharp angle of the splenic artery with the celiac axis and the rhythmic contraction by the sinusoidal splenic architecture were speculated as limiting factors of metastasis [9]. Spleen is the second largest organ of the reticuloendothelial system with profuse monocytes, immunoglobulin synthesis, and opsonin production. Therefore, immune surveillance appears to potently inhibit tumor cell proliferation [9]. On the other hand, intense destruction of malignant cells in spleen may limit the development of splenic metastasis as supported by the experimental evidences [16]. The absence of afferent lymphatics to the spleen is considered to be another reason for such low incidence of splenic metastasis [12].
Another considerable point is the potential relationship between the primary tumor site and isolated splenic metastasis. In fact, in 12 of the 14 reported patients including the present case, the primary tumor was in the left colon ( 5 in sigmoid colon, 3 in descending colon, 2 in rectum, and 2 at left flexure), whereas it was in cecum and ascending colon in the remaining two. As previously stated by Avesani et al. [11], this observation may support the theory by Indudhara et al. [6]; a possible retrograde spread to the spleen via the inferior mesenteric vein. However, this explanation has to stay theoretical; because, there is unfortunately no current imaging study to be able to show this type spread in an individual basis.

Diagnosis of splenic metastasis as well as other remote organ metastasis from colorectal cancer can be made by help of many current imaging studies such as US, CT or MRI. However, FDG-PET scanning provides a better detection of metastatic deposits, especially when they are too small to be shown by other radiological studies in the setting of postoperatively rising CEA level. In addition, PETCT fusion may specifically suggest that the mass seen by the CT is tumoral in nature. On the other hand, solitary status of metastasis can be demonstrated and predicted preoperatively by PET-CT fusion scanning as in the present case. Therefore, the indication of splenectomy can 


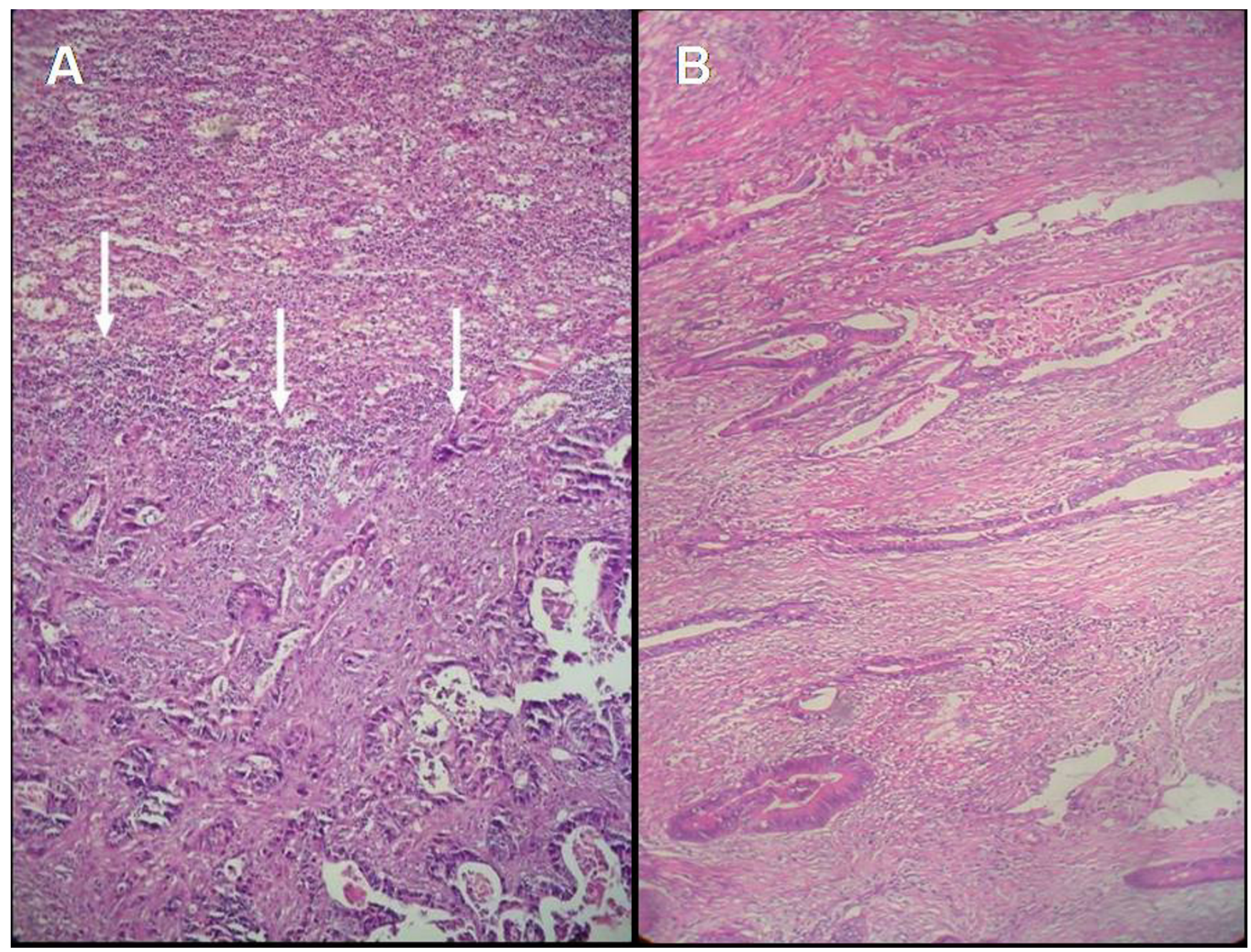

\section{Figure 4}

Histological section of the splenic tumor showing glandular pattern consistent with metastasis from colonic adenocarcinoma (arrows show the tumor within the splenic parenchyma), X100, Hematoxylin +Eosin (A). Histologic section of the primary colonic tumor located at the sigmoid colon with glandular pattern, XI00, Hematoxylin +Eosin (B).

be justified in such cases since the presence of multiple remote site metastases usually exterminates the chance of surgical intervention.

Long-term survival rate following splenectomy in patients with solitary splenic metastasis from colorectal cancer is still unknown. However, the limited data extracted from the case reports in the literature indicate that these patients may survive up to 7 years $[8,12,13]$. The present case is disease-free during the one-year of follow up.

\section{Conclusion}

Isolated splenic metastasis is a rare finding in the followup of colorectal cancer patients and long-term survival can be achieved with splenectomy.

\section{Abbreviations}

CEA: carcinoembryonic antigen

${ }^{18}$ FDG-PET: ${ }^{18}$ Fluorodeoxyglucose - positron emission tomography

PET-CT: positron emission tomography - computed tomography

US: ultrasound

CT: computed tomography

MRI: magnetic resonance imaging 


\section{Competing interests}

The author(s) declare that they have no competing interests.

\section{Authors' contributions}

RG performed the operations and prepared the manuscript. FA and GK performed the histopathologic evaluations. NT participated in the redaction of the article. All authors read and approved the final manuscript.

\section{Acknowledgements}

Written consent was obtained from the patient for publication of the study.

\section{References}

I. Dunbar WH, Beahrs, Morlock CG: Solitary splenic metastasis incidental to rectal carcinoma: report of a case. Mayo Clin Proc 1969, 44:40-45.

2. Waller RM III, Fajman WA: An unusual cause of an isolated, focal splenic defect demonstrated by liver-spleen scintigraphy. Clin Nucl Med 1982, 7:5-6.

3. Slavin JD Jr, Matthews J, Spencer RP: Splenectomy for splenic metastasis from carcinoma of the colon. Clin Nucl Med 1986, I I:491-492.

4. Capizzi PJ, Allen KB, Amerson JR, Skandalakis JE: Isolated splenic metastasis from rectal carcinoma. South Med J I992, 85: $1003-1005$.

5. Thomas SM, Fitzgerald JB, Pollock RE, Evans DB: Isolated splenic metastases from colon carcinoma. Eur J Surg Oncol 1993, 19:485-490.

6. Indudhara R, Vogt D, Levin HS, Church J: Isolated splenic metastases from colon cancer. South Med J 1997, 90:633-636.

7. Mainprize KS, Berry AR: Solitary splenic metastasis from colorectal carcinoma. Br J Surg 1997, 84:70.

8. Weathers BK, Modesto VL, Gordon D: Isolated splenic metastasis from colorectal carcinoma: report of a case and review of the literature. Dis Colon Rectum 1999, 42: I345-I348.

9. Kim JC, Jeong CS, Kim HC, Yu CS, Kang GH, Lee MG: Isolated splenic metastasis from colorectal carcinoma: a case report. J Korean Med Sci 2000, I 5:355-358.

10. Place RJ: Isolated colon cancer metastasis to the spleen. Am Surg 200I, 67:454-457.

II. Avesani EC, Cioffi U, De Simone M, Botti F, Carrara A, Ferrero S: Synchronous isolated splenic metastasis from colon carcinoma. Am J Clin Oncol 2001, 24:3 II-3I2.

12. Okuyama $\mathrm{T}$, Oya M, Ishikawa $\mathrm{H}$ : Isolated splenic metastasis of sigmoid colon cancer: a case report. Jpn J Clin Oncol 200I, 3 I:34 I-345.

13. Cavallaro A, Modugno P, Specchia M, Pontenza AE, Loschiavo V, Colli $\mathrm{R}$, Lauriola $\mathrm{L}$, Barone $\mathrm{C}$ : Isolated splenic metastasis from colon cancer. J Exp Clin Cancer Res 2004, 23:|43-146.

14. Berge T: Splenic metastases. Acta Pathol Microbiol Scand 1974, 82:499-506

15. Skandalakis LJ, Gray SW, Skandalakis JE: Splenic realities and curiosities. Prob Gen Surg 1990, 7:28-32.

16. Eichner ER: Splenic function, normal, too much and too little. Am J Med 1979, 66:31 I-320.
Publish with Biomed Central and every scientist can read your work free of charge

"BioMed Central will be the most significant development for disseminating the results of biomedical research in our lifetime. "

Sir Paul Nurse, Cancer Research UK

Your research papers will be:

- available free of charge to the entire biomedical community

- peer reviewed and published immediately upon acceptance

- cited in PubMed and archived on PubMed Central

- yours - you keep the copyright
BioMedcentral 